\title{
Predictors of mortality in patients with carbapenem-resistant Klebsiella pneumoniae infection: a meta-analysis and a systematic review
}

\author{
Yiyi Qian" ${ }^{1 \#}$, Yingmin $\mathrm{Bi}^{2 \#}$, Shuang $\mathrm{Liu}^{2 \#}$, Xiangyu $\mathrm{Li}^{3}$, Shuaiyue Dong ${ }^{4}$, Mohan $\mathrm{Ju}^{2}$ \\ ${ }^{1}$ Department of Infectious Diseases, Zhongshan Hospital, Fudan University, Shanghai, China; ${ }^{2}$ Institute of Antibiotics, Huashan Hospital North, \\ Fudan University, Shanghai, China; ${ }^{3}$ Department of Clinical Laboratory, Huashan Hospital North, Fudan University, Shanghai, China; ${ }^{4}$ Department \\ of Emergency, Huashan Hospital North, Fudan University, Shanghai, China \\ Contributions: (I) Conception and design: Y Qian, M Ju; (II) Administrative support: M Ju; (III) Provision of study materials or patients: S Liu, \\ S Dong, X Li; (IV) Collection and assembly of data: Y Bi, Y Qian, M Ju; (V) Data analysis and interpretation: Y Bi, Y Qian, M Ju; (VI) Manuscript \\ writing: All authors; (VII) Final approval of manuscript: All authors. \\ "These authors contributed equally to this work. \\ Correspondence to: Mohan Ju. Luxiang Road 108th, District Baoshan, Shanghai, China. Email: 08301010203@fudan.edu.cn.
}

Background: Cases of carbapenem-resistant Klebsiella pneumoniae infection have been increasing. Patients
with carbapenem-resistant Klebsiella pneumoniae infection have a poor prognosis and a high mortality
rate. Identification of potential risk factors associated with carbapenem-resistant Klebsiella pneumoniae
infection-related mortality may help improve patient outcomes.
Methods: Embase, PubMed, and the Cochrane Library databases were searched to identify articles
describing predictors of mortality in patients with carbapenem-resistant Klebsiella pneumoniae infection. The
quality of articles was assessed with the Newcastle-Ottawa Scale score (NOS). Review Manager was used for
statistical analyses.

Results: Twenty-seven observational studies were included in the analysis. Factors associated with higher mortality were septic shock [odds ratio (OR): 4.41, 95\% CI: 3.17-6.15], congestive heart failure (OR: 2.65, 95\% CI: 1.71-4.13), chronic obstructive pulmonary disease (COPD; OR: 2.43, 95\% CI: 1.87-3.15), chronic kidney disease (CKD; OR: 1.78, 95\% CI: 1.43-2.22), diabetes mellitus (OR: 1.41, 95\% CI: 1.16-1.72), mechanical ventilation (OR: 1.65, 95\% CI: 1.25-2.18), and inappropriate empirical antimicrobial treatment (OR: 1.25, 95\% CI: 1.03-1.52). The average Acute Physiology and Chronic Health Evaluation (APACHE) II score at the time of diagnosis of carbapenem-resistant Klebsiella pneumoniae infection was considerably higher in patients who did not survive than in those who survived (weighted mean difference: $5.86,95 \%$ CI: 2.46-9.26).

Discussion: Patient condition, timing appropriate antimicrobial treatment, and disease severity according to the APACHE II score are the most important risk factors for death in patients with carbapenem-resistant Klebsiella pneumoniae infection. Our finding may help predict patients' outcomes and improve management for them.

Registration number 20210417 EuEGX/INPLASY2020100037.

Keywords: Carbapenem-resistant Klebsiella pneumoniae; antibiotic resistance; mortality; risk factors

Submitted Feb 06, 2021. Accepted for publication May 18, 2021.

doi: 10.21037/apm-21-338

View this article at: https://dx.doi.org/10.21037/apm-21-338 


\section{Introduction}

As an important opportunistic pathogen, Klebsiella pneumoniae can cause nosocomial infections [such as hospital-acquired or ventilator-associated pneumonia (VAP)], urinary tract infections, and bacteremia among patients with critical illness (1). Carbapenems are one of the most widely used antibacterial drugs for the treatment of severe infections caused by K. pneumoniae. However, the number of cases of carbapenem-resistant $K$. pneumoniae (CRKP) infection has been increasing and has outnumbered the number of cases carbapenem-susceptible $K$. pneumoniae (CSKP) infections in recent years. The proportion of $K$. pneumoniae isolates resistant to carbapenems increased from $2.9 \%$ in 2005 to more than $25 \%$ in 2018 in China (2). Because of the limited range of antibiotics available, patients infected with CRKP have a poor prognosis and a high mortality rate. CRKP infection has become a serious clinical challenge and has attracted the attention of medical institutions worldwide.

Previous studies have found a higher mortality rate in patients with CRKP infections than in those with CSKP infections (3). It is thus essential to identify potential risk factors associated with the mortality of CRKP infection, which may help improve patients' outcomes. Some risk factors, such as intensive care unit (ICU) stays, inappropriate antimicrobial treatment, and higher Acute Physiology and Chronic Health Evaluation (APACHE) II scores, have been identified $(4,5)$. The results of several studies remain controversial. For example, though most relevant studies reveal that the indwelling of a central venous catheter is positively associated with fatality, others draw the opposite conclusion (6-9). Therefore, a meta-analysis was conducted to evaluate the predictors of mortality in patients infected with CRKP. We present the following article in accordance with the PRISMA reporting checklist (available at https:// dx.doi.org/10.21037/apm-21-338).

\section{Methods}

\section{Search strategy}

Two independent examiners (MHJ and YYQ) conducted a comprehensive search in the PubMed, EMBASE, and Cochrane Library databases from their inception to July 31, 2020 for relevant articles. The search strategy used the following keywords: ("Klebsiella pneumoniae" OR "Klebsiella" OR "K. pneumoniae") AND ("resistance" OR "resistant") AND ("carbapenem" OR "meropenem" OR "imipenem"
OR "doripenem" OR "ertapenem") AND ("mortality" OR "lethality" OR "fatality" OR "prognosis" OR "survival" OR "predictor"). Furthermore, reference lists cited by eligible retrieved articles were also manually retrieved to maximize inclusion of studies. Only articles written in English were reviewed.

\section{Selection criteria}

This meta-analysis included observational studies reporting mortality and associated risk factors of patients with CRKP infection. Carbapenem resistance referred to resistance to carbapenems, including meropenem, imipenem, ertapenem, or doripenem. The primary outcome was mortality. After review by 2 independent examiners, non-original articles, such as reviews, meta-analyses, case reports, in vitro or experimental animal studies, or pediatric studies were not included. Studies in which CRKP status (infection/colonization) was not clarified were also excluded. The protocol for this systematic review was registered on INPLASY (Unique ID 2020100037) and is available in full on inplasy.com (https://doi.org/10.37766/ inplasy2020.10.0037).

\section{Quality assessment and data extraction}

The methodological quality of articles included was assessed with the Newcastle-Ottawa Scale score (NOS) (10). Two independent examiners (MHJ and YYQ) performed the NOS assessment for each study. Inconsistencies between the 2 investigators were extensively discussed until agreement was achieved. Studies with an NOS score of at least 5 underwent further analysis, while others were excluded because of the potential high risk of bias. Two investigators (MHJ and YYQ) independently extracted the relevant data from each eligible article, including authors, date of publication, location, study design and period, sample size, patient population characteristics (such as age, sex, infection site, comorbidities), severity of diseases, microbiologic data, invasive procedures, ICU admission, and treatment variables. Variables examined in less than 2 eligible studies were excluded.

\section{Statistical analysis}

Review Manager (version 5.3 software) was used for statistical analyses. Heterogeneity was tested with the Q statistic (significant when $\mathrm{P}<0.10$ ) and the extent of heterogeneity 


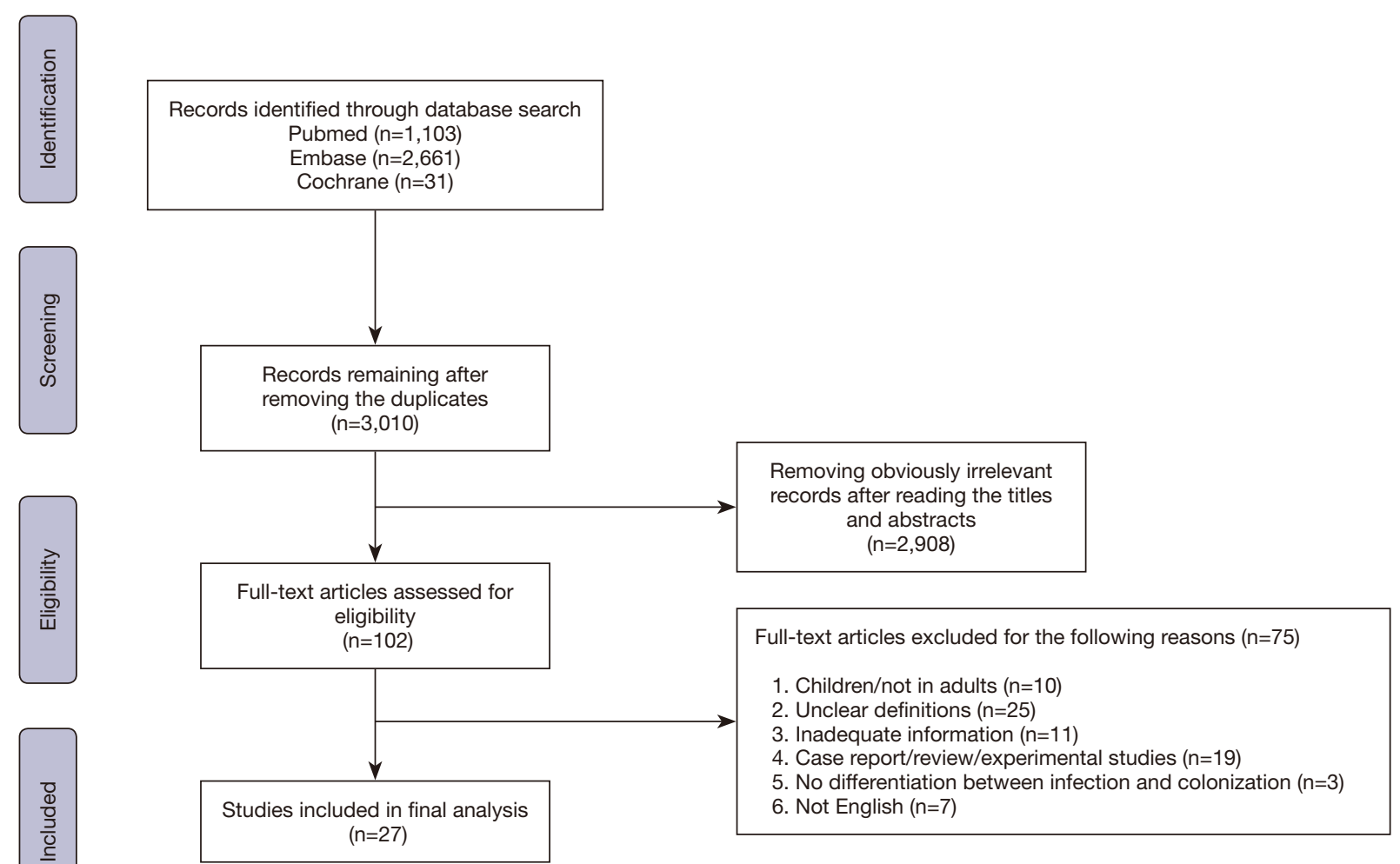

Figure 1 Flow diagram of included studies.

was quantified with the $\mathrm{I}^{2}$ statistic. $\mathrm{I}^{2}>50 \%$ was interpreted as substantial and significant heterogeneity or inconsistency. Pooled odds ratios (ORs) and 95\% CIs were calculated to express binary outcome results, while the weighted mean difference (WMD) and 95\% CIs were used to express continuous outcome results $(11,12)$. Sensitivity analysis on the literature included was done by omitting each study one by one at a time in the process of meta-analysis to inspect the change of merging effect, thus demonstrating the stability and accuracy of the outcome. Publication bias was identified by funnel plots.

\section{Results}

\section{Results of study inclusion}

The literature search identified a total of 3,795 publications. After duplicates were removed, 3,010 articles were screened. After reviewing abstracts and titles for obvious irrelevancy, 2,908 were ruled out. After reviewing the full texts, we excluded another 75 studies according to the eligibility criteria. Twenty-seven studies were included in the final analysis. The process of article selection is shown in Figure 1.

\section{Study characteristics}

The characteristics of the 27 studies included are summarized in Table 1. Of the 27 studies, 10 were multicenter studies and 17 were single-center studies. Most (23/27) had a retrospective design. The studies were from 7 countries/ regions, including China $(n=6)$, the USA $(n=4)$, Italy $(n=8)$, Spain $(n=3)$, and Greece $(n=4)$, all published between 2008 and 2020. Sample sizes ranged from 14 to 661, and 3,699 patients with CRKP infection were included in the systematic review in total. Among them, 1,280 (34.6\%) were reported deaths. Various types of infection were included, with bloodstream infection (BSI) being the most common. Eighteen studies focused on patients with BSI, sepsis, or septic shock caused by CRKP. Eight studies focused on patients with all types of infections, and one study focused on patients with VAP.

\section{Predictors of death in patients with CRKP}

Potential risk factors associated with death in patients with CRKP infection were analyzed (Table 2). Notably, 


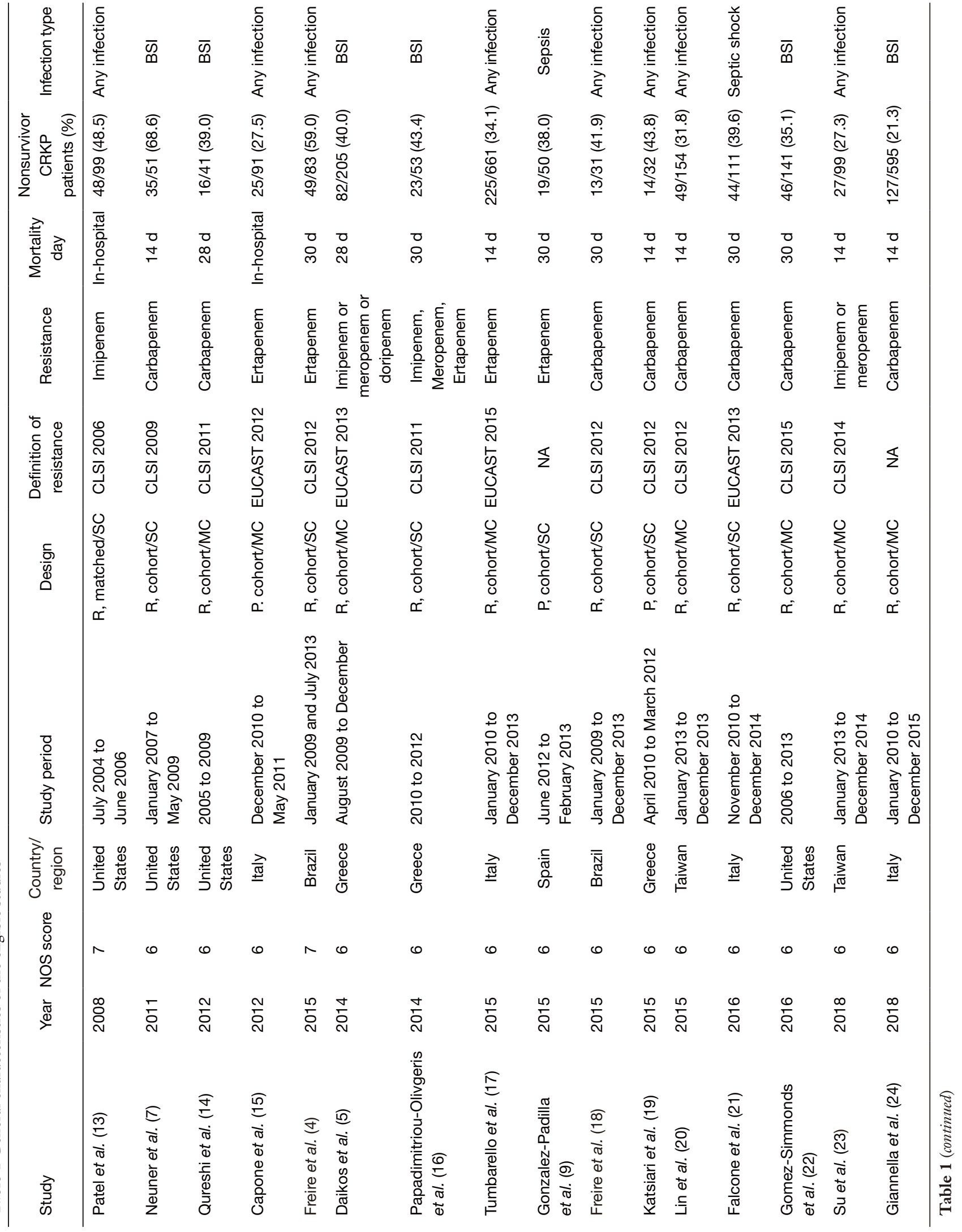




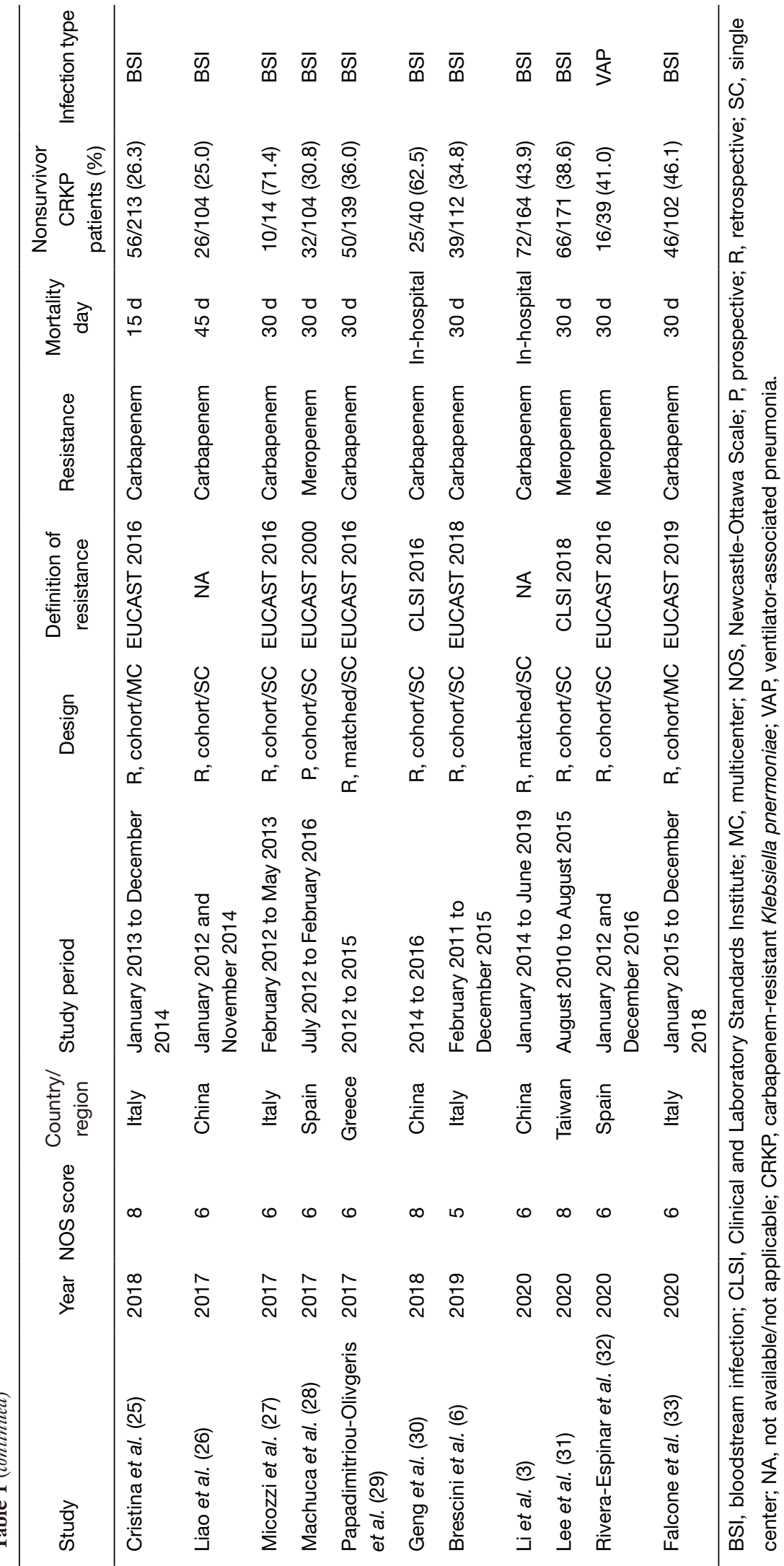


only factors mentioned in at least 3 studies were included. As shown in Table 2, patients' comorbidities at admission, including presentation with septic shock (OR: 4.41; 95\% CI: 3.17-6.15) (Figure 2), congestive heart failure (OR: 2.65, 95\% CI: 1.71-4.13), chronic obstructive pulmonary disease (COPD; OR: 2.43, 95\% CI: 1.87-3.15), chronic kidney disease (CKD; OR: 1.78, 95\% CI: 1.43-2.22), diabetes mellitus (OR: 1.41, 95\% CI: 1.16-1.72), and mechanical ventilation (OR: 1.65, 95\% CI: 1.25-2.18), were considered to increase mortality. To our surprise, comorbidities involving an immunocompromised status, such as neutropenia, transplantation, solid malignancies, immunosuppressant use, and chemotherapy or radiotherapy, were not risk factors for death. However, inappropriate empirical antimicrobial treatment (OR: 1.25, 95\% CI: $1.03-$ 1.52) may lead to a higher mortality rate, while optimized targeted treatment (OR: 0.38, 95\% CI: 0.25-0.57) improved patients' survival. Interestingly, our results show that a previous surgical history (OR: $0.78,95 \%$ CI: $0.61-0.99$ ) was associated with a better clinical outcome.

\section{Continuous risk factors of death in patients with CRKP}

Several important continuous variables have been assessed for the association with mortality in patients with CRKP (Table 3). As shown, quantitative analysis with a fixed- or random-effects model indicated that older age, a longer hospital stay before infection, and symptom severity (evaluated with APACHE II score) were significantly correlated with higher mortality. Notably, the APACHE II score, especially upon diagnosis of CRKP infection, was much higher in the non-survival group than in the survival group (WMD, 5.86; 95\% CI: 2.46-9.26).

\section{Sensitivity analysis}

In this research, the sensitivity analysis was performed through eliminating each included study one by one. We found that the OR value, $95 \% \mathrm{CI}$ and $\mathrm{P}$ value after omission were very close in most of the risk factors to the results when the study was not omitted. Nevertheless, when we removed the study of Tumbarello (17), the ORs and the corresponding $95 \% \mathrm{CI}$ for solid malignancies changed from 1.21 (95\% CI: $0.76-1.93$ ) to 1.57 (95\% CI: $1.06-2.32$ ), the ORs and the corresponding $95 \%$ CIs for cardiovascular diseases changed to 2.82 (95\% CI: 0.97-8.21). The results and statistically significant difference changed for the solid malignancies factor and cardiovascular diseases factor.

\section{Publication bias evaluation}

In this research, we accessed the publication bias for each related risk factor by funnel plot and in each funnel plot we failed to find any distinct asymmetry which means the bias was generally balanced. The results showed that the two sides were basically symmetrical, and individual studies were all in the $95 \%$ CI, suggesting that there was a small probability of publication bias in the included study. One representative funnel plot to assess publication bias for Diabetes mellitus is demonstrated in Figure 3.

\section{Discussion}

CRKP infection has been increasing over the years and is associated with a high mortality rate. It is critical to predict and improve the outcome of these patients. Here, we performed a meta-analysis of the existing literature to identify risk factors associated with mortality in patients with CRKP infection, thus providing possible suggestions on the appropriate clinical decisions for physicians.

Comorbidities, including the presentation of congestive heart failure, COPD, renal failure, CKD, and diabetes mellitus, are associated with increased mortality, which may be expected. patients with CRKP infection with these comorbidities should be closely monitored as they tend to have a poorer prognosis.

Our results gave a clear demonstration that appropriate antimicrobial therapy can increase the survival rate in patients with CRKP infection. Firstly, inappropriate empirical antimicrobial treatment increased the pooled mortality of 2,045 patients with CRKP infection, which underlines the importance of bacterial resistance monitoring in healthcare facilities and the qualified use of antimicrobials by physicians. In the absence of bacterial identification or drug sensitivity results in clinical settings with a high incidence of CRKP, a more aggressive initial regimen involving anti-CRKP antimicrobials, such as colistin, tigecycline, and ceftazidime/avibactam, should be launched as soon as possible under the supervision of infectious disease specialists. However, optimized targeted treatment substantially increased the survival of 1,716 patients across 18 studies. Therefore, in clinical practice, after culture and drug sensitivity tests results are available, physicians should adjust therapy to an optimized treatment immediately. Some PCR-based molecular methods, due to their shorter turnaround time compared to drug sensitivity tests, could be used to identify organisms, and detect certain 
Table 2 Risk factors for mortality in patients with CRKP upon diagnosis

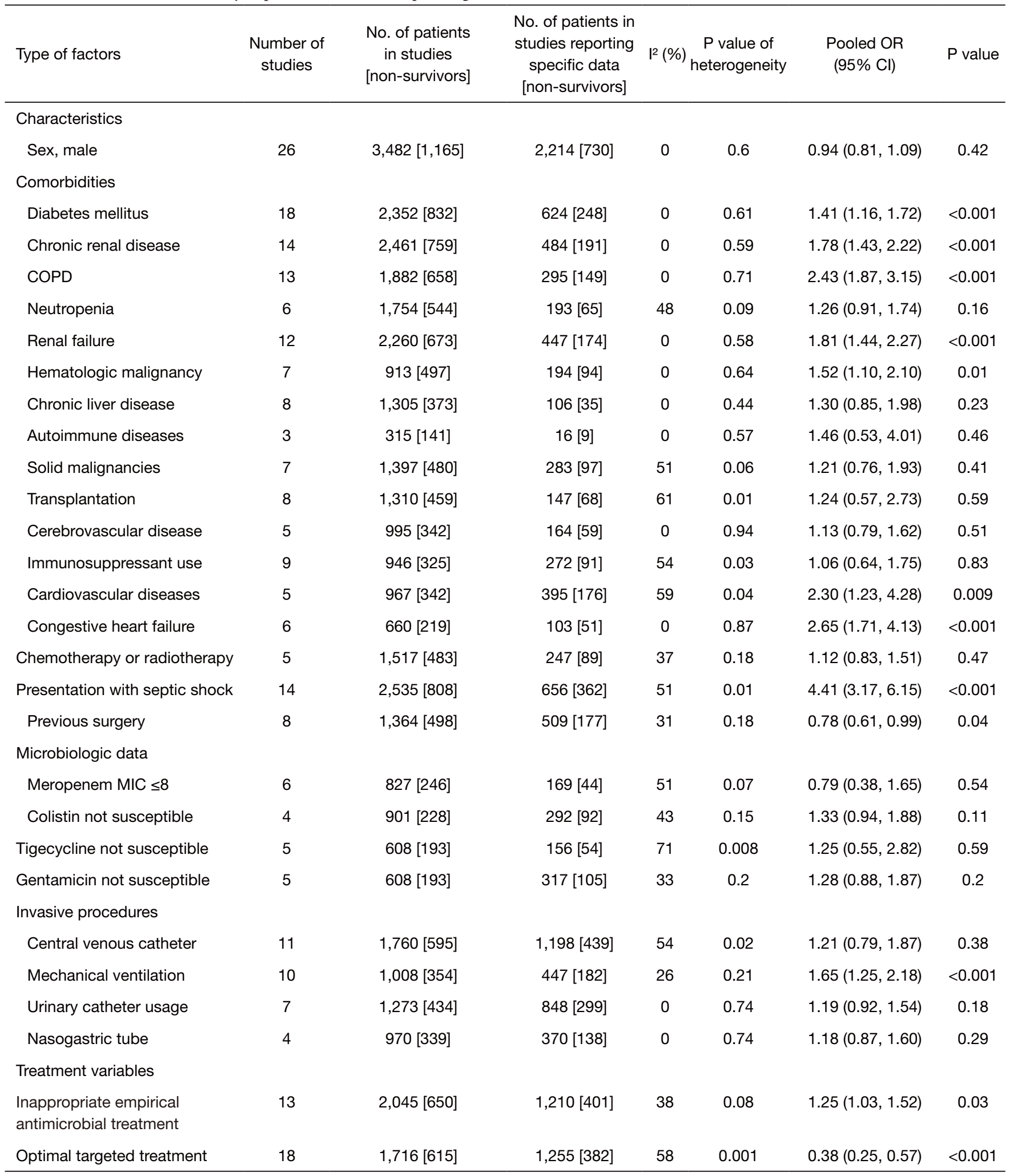

$\mathrm{Cl}$, confidence interval; CRKP, carbapenem-resistant Klebsiella pneumoniae; OR, odds ratio. 


\begin{tabular}{|c|c|c|c|c|c|}
\hline \multirow[b]{2}{*}{ Study or Subgroup } & \multicolumn{2}{|c|}{ Experimental } & \multicolumn{2}{|c|}{ Control } & \multirow[b]{2}{*}{ Weight } \\
\hline & Events & Total & Events & Total & \\
\hline Brescini 2019 & 26 & 39 & 14 & 73 & $7.6 \%$ \\
\hline Cristina 2017 & 23 & 56 & 19 & 157 & $9.3 \%$ \\
\hline Daikos 2014 & 24 & 82 & 15 & 123 & $9.2 \%$ \\
\hline Falcone 2020 & 22 & 46 & 18 & 56 & $8.3 \%$ \\
\hline Geng 2018 & 22 & 25 & 5 & 15 & $3.4 \%$ \\
\hline Giannella 2017 & 52 & 127 & 60 & 468 & $12.6 \%$ \\
\hline Gomez-Simmonds 2016 & 24 & 46 & 19 & 95 & $8.7 \%$ \\
\hline M. Katsiari 2015 & 14 & 14 & 11 & 18 & $1.2 \%$ \\
\hline M. Papadimitriou-Olivgeris 2014 & 15 & 23 & 3 & 30 & $3.9 \%$ \\
\hline M. Papadimitriou-Olivgeris 2017 & 43 & 50 & 31 & 89 & $7.3 \%$ \\
\hline Machuca 2017 & 17 & 32 & 31 & 72 & $8.0 \%$ \\
\hline Rivera-Espinar 2020 & 15 & 16 & 17 & 23 & $2.0 \%$ \\
\hline Su 2017 & 8 & 27 & 8 & 72 & $5.8 \%$ \\
\hline Tumbarello 2015 & 57 & 225 & 43 & 436 & $12.8 \%$ \\
\hline Total $(95 \% \mathrm{Cl})$ & & 808 & & 1727 & $100.0 \%$ \\
\hline Total events & 362 & & 294 & & \\
\hline $\begin{array}{l}\text { Heterogeneity: } \mathrm{Tau}^{2}=0.18 ; \mathrm{Ch}^{2} \\
\text { Test for overall effect: } Z=8.77 \text { ( }\end{array}$ & $\begin{array}{l}6.61, \mathrm{df} \\
.00001)\end{array}$ & $3(\mathrm{P}$ & .01); & & \\
\hline
\end{tabular}

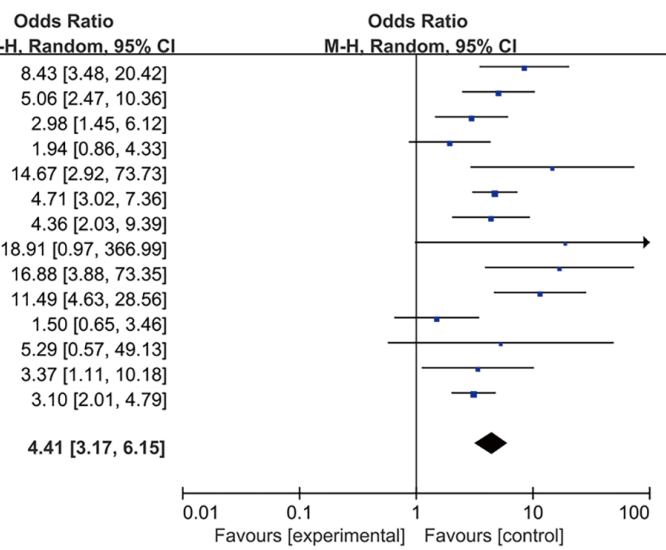

Figure 2 Meta-analysis on the forest plots of presentation of septic shock.

Table 3 Continuous variables and risk for mortality in patients infected with CRKP

\begin{tabular}{|c|c|c|c|c|c|c|}
\hline Continuous variable & $\begin{array}{l}\text { No. of } \\
\text { studies }\end{array}$ & $\begin{array}{l}\text { No. of patients in studies } \\
\text { reporting specific data } \\
\text { [non-survivors] }\end{array}$ & $\mathrm{I}^{2}(\%)$ & $\begin{array}{c}\mathrm{P} \text { value of } \\
\text { heterogeneity }\end{array}$ & WMD $(95 \% \mathrm{Cl})$ & $P$ value \\
\hline Hospital stay before infection (days) & 7 & 702 [268] & 0 & 0.78 & $2.27(0.28,4.25)$ & 0.03 \\
\hline $\begin{array}{l}\text { APACHE II score upon diagnosis of } \\
\text { CRKP infection }\end{array}$ & 6 & $1,114[311]$ & 85 & $<0.0001$ & $5.86(2.46,9.26)$ & $<0.001$ \\
\hline APACHE II score at admission to ICU & 6 & 472 [205] & 0 & 0.95 & $2.51(1.21,3.81)$ & $<0.001$ \\
\hline
\end{tabular}

APACHE II, Acute Physiology and Chronic Health Evaluation II score; CRKP, carbapenem-resistant Klebsiella pneumoniae; ICU, intensive care unit; WMD, weighted mean difference.

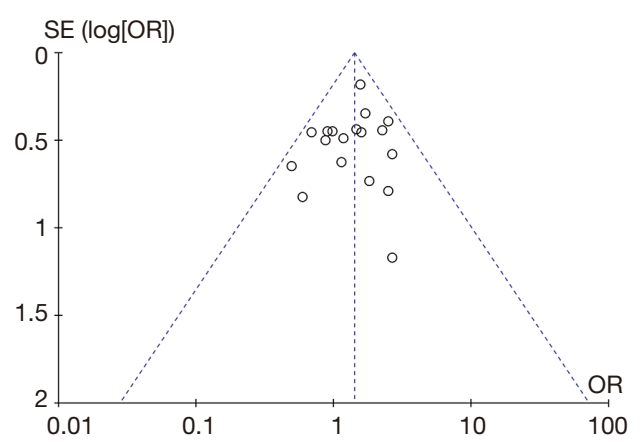

Figure 3 Funnel plot to assess publication bias for diabetes mellitus. OR, odds ratio.

drug resistance genes, such as those encoding KPC, NDM, and VIM, thus shortening the duration to optimal targeted treatment and improving patients' outcomes (34).

At the same time, attention should be paid to the patient's condition, including underlying diseases. Our results suggested that dysfunction of certain organs (e.g., congestive heart failure, COPD, chronic renal disease, diabetes mellitus) may serve as predictors of a poor prognosis. In addition, the presentation of septic shock increased the pooled mortality of patients with CRKP infection more than fourfold, which is quite understandable considering that septic shock represents a rather severe condition of infection. However, in this study, an immunocompromised status, as noted by neutropenia or immunosuppressant use, was not associated with increased mortality.

Our study shows that a history of previous surgery served as a protective factor for patients with CRKP infection. This could be explained by the fact that patients who were able to undergo surgery tended to have a better overall health status and fewer comorbidities.

Our analysis showed that, among the continuous variables, older age and higher APACHE II score were the main predictors of CRKP infection mortality. The impact of age on outcome is quite understandable, since older 
patients may have more underlying diseases. In addition, the APACHE II score proved to be an important and useful tool for the evaluation of disease severity and the prediction of outcomes in patients with CRKP infection, which has also been shown in the analysis of other pathogens, such as carbapenem-resistant Acinetobacter baumannii (35). However, our study shows that APACHE II score upon diagnosis of CRKP infection may have a greater impact on mortality than at ICU admission. This indicates that the objective assessment of disease severity using the APACHE II score should be conducted at an earlier stage. Therefore, more aggressive management could be taken in advance for patients with a potentially worse outcome, thus improving their chance of survival.

\section{Limitations}

There are limitations of this study. First, most of the studies included in this analysis were retrospective observational studies, which may be susceptible to selection bias and thus should be considered as lower-evidence studies. Further prospectively designed studies are required. Second, only studies written in English were included, which may introduce an additional level of bias.

\section{Conclusions}

Age, patient condition, timing and appropriate antimicrobial treatment, and disease severity evaluated by the APACHE II score are the most important predictors of mortality in patients with CRKP infection. These findings may help physicians to predict outcomes in patients with CRKP infection and help to improve the management of these patients.

\section{Acknowledgments}

We would like to thank Jia Tang for her precious suggestion on the idea of this study.

Funding: This work was supported by the project of Huashan Hospital North (HSBY2019007) and grants from the Shanghai Municipal Planning Commission of Science and Research Fund (20204Y0441).

\section{Footnote}

Reporting Checklist: The authors have completed the PRISMA reporting checklist. Available at https://dx.doi. org/10.21037/apm-21-338
Peer Review File: Available at https://dx.doi.org/10.21037/ apm-21-338

Conflicts of Interest: All authors have completed the ICMJE uniform disclosure form (available at https:// dx.doi.org/10.21037/apm-21-338) and report the funding support from the project of Huashan Hospital North (HSBY2019007) and grants from the Shanghai Municipal Planning Commission of Science and Research Fund (20204Y0441). The authors have no other conflicts of interest to declare.

Ethical Statement: The authors are accountable for all aspects of the work in ensuring that questions related to the accuracy or integrity of any part of the work are appropriately investigated and resolved.

Open Access Statement: This is an Open Access article distributed in accordance with the Creative Commons Attribution-NonCommercial-NoDerivs 4.0 International License (CC BY-NC-ND 4.0), which permits the noncommercial replication and distribution of the article with the strict proviso that no changes or edits are made and the original work is properly cited (including links to both the formal publication through the relevant DOI and the license). See: https://creativecommons.org/licenses/by-nc-nd/4.0/.

\section{References}

1. Tang M, Kong X, Hao J, et al. Epidemiological Characteristics and Formation Mechanisms of MultidrugResistant Hypervirulent Klebsiella pneumoniae. Front Microbiol 2020;11:581543.

2. Hu F, Guo Y, Yang Y, et al. Resistance reported from China antimicrobial surveillance network (CHINET) in 2018. Eur J Clin Microbiol Infect Dis 2019;38:2275-81.

3. $\mathrm{Li} \mathrm{Y,} \mathrm{Li} \mathrm{J,} \mathrm{Hu} \mathrm{T,} \mathrm{et} \mathrm{al.} \mathrm{Five-year} \mathrm{change} \mathrm{of} \mathrm{prevalence} \mathrm{and}$ risk factors for infection and mortality of carbapenemresistant Klebsiella pneumoniae bloodstream infection in a tertiary hospital in North China. Antimicrob Resist Infect Control 2020;9:79.

4. Freire MP, Pierrotti LC, Filho HH, et al. Infection with Klebsiella pneumoniae carbapenemase (KPC)-producing Klebsiella pneumoniae in cancer patients. Eur J Clin Microbiol Infect Dis 2015;34:277-86.

5. Daikos GL, Tsaousi S, Tzouvelekis LS, et al. Carbapenemase-producing Klebsiella pneumoniae bloodstream infections: lowering mortality by antibiotic 
combination schemes and the role of carbapenems. Antimicrob Agents Chemother 2014;58:2322-8.

6. Brescini L, Morroni G, Valeriani C, et al. Clinical and epidemiological characteristics of KPC-producing Klebsiella pneumoniae from bloodstream infections in a tertiary referral center in Italy. BMC Infect Dis 2019;19:611.

7. Neuner EA, Yeh JY, Hall GS, et al. Treatment and outcomes in carbapenem-resistant Klebsiella pneumoniae bloodstream infections. Diagn Microbiol Infect Dis 2011;69:357-62.

8. Zarkotou O, Pournaras S, Tselioti P, et al. Predictors of mortality in patients with bloodstream infections caused by KPC-producing Klebsiella pneumoniae and impact of appropriate antimicrobial treatment. Clin Microbiol Infect 2011;17:1798-803.

9. Gonzalez-Padilla M, Torre-Cisneros J, Rivera-Espinar F, et al. Gentamicin therapy for sepsis due to carbapenemresistant and colistin-resistant Klebsiella pneumoniae. J Antimicrob Chemother 2015;70:905-13.

10. Stang A. Critical evaluation of the Newcastle-Ottawa scale for the assessment of the quality of nonrandomized studies in meta-analyses. Eur J Epidemiol 2010;25:603-5.

11. Luo D, Wan X, Liu J, et al. Optimally estimating the sample mean from the sample size, median, mid-range, and/or mid-quartile range. Stat Methods Med Res 2018;27:1785-805.

12. Wan X, Wang W, Liu J, et al. Estimating the sample mean and standard deviation from the sample size, median, range and/or interquartile range. BMC Med Res Methodol 2014;14:135.

13. Patel G, Huprikar S, Factor SH, et al. Outcomes of carbapenem-resistant Klebsiella pneumoniae infection and the impact of antimicrobial and adjunctive therapies. Infect Control Hosp Epidemiol 2008;29:1099-106.

14. Qureshi ZA, Paterson DL, Potoski BA, et al. Treatment outcome of bacteremia due to KPC-producing Klebsiella pneumoniae: superiority of combination antimicrobial regimens. Antimicrob Agents Chemother 2012;56:2108-13.

15. Capone A, Giannella M, Fortini D, et al. High rate of colistin resistance among patients with carbapenemresistant Klebsiella pneumoniae infection accounts for an excess of mortality. Clin Microbiol Infect 2013;19:E23-30.

16. Papadimitriou-Olivgeris $M$, Marangos M, Christofidou $\mathrm{M}$, et al. Risk factors for infection and predictors of mortality among patients with KPC-producing Klebsiella pneumoniae bloodstream infections in the intensive care unit. Scand J Infect Dis 2014;46:642-8.
17. Tumbarello M, Trecarichi EM, De Rosa FG, et al. Infections caused by KPC-producing Klebsiella pneumoniae: differences in therapy and mortality in a multicentre study. J Antimicrob Chemother 2015;70:2133-43.

18. Freire MP, Abdala E, Moura ML, et al. Risk factors and outcome of infections with Klebsiella pneumoniae carbapenemase-producing K. pneumoniae in kidney transplant recipients. Infection 2015;43:315-23.

19. Katsiari M, Panagiota G, Likousi S, et al. Carbapenemresistant Klebsiella pneumoniae infections in a Greek intensive care unit: Molecular characterisation and treatment challenges. J Glob Antimicrob Resist 2015;3:123-7.

20. Lin Y'T, Chuang C, Su CF, et al. Efficacy of Appropriate Antimicrobial Therapy on the Survival of Patients With Carbapenem Nonsusceptible Klebsiella Pneumoniae Infection: A Multicenter Study in Taiwan. Medicine (Baltimore) 2015;94:e1405.

21. Falcone M, Russo A, Iacovelli A, et al. Predictors of outcome in ICU patients with septic shock caused by Klebsiella pneumoniae carbapenemase-producing K. pneumoniae. Clin Microbiol Infect 2016;22:444-50.

22. Gomez-Simmonds A, Nelson B, Eiras DP, et al. Combination Regimens for Treatment of CarbapenemResistant Klebsiella pneumoniae Bloodstream Infections. Antimicrob Agents Chemother 2016;60:3601-7.

23. Su CF, Chuang C, Lin YT, et al. Treatment outcome of non-carbapenemase-producing carbapenem-resistant Klebsiella pneumoniae infections: a multicenter study in Taiwan. Eur J Clin Microbiol Infect Dis 2018;37:651-9.

24. Giannella M, Trecarichi EM, Giacobbe DR, et al. Effect of combination therapy containing a high-dose carbapenem on mortality in patients with carbapenemresistant Klebsiella pneumoniae bloodstream infection. Int J Antimicrob Agents 2018;51:244-8.

25. Cristina ML, Alicino C, Sartini M, et al. Epidemiology, management, and outcome of carbapenem-resistant Klebsiella pneumoniae bloodstream infections in hospitals within the same endemic metropolitan area. J Infect Public Health 2018;11:171-7.

26. Liao Y, Hu GH, Xu YF, et al. Retrospective analysis of fosfomycin combinational therapy for sepsis caused by carbapenem-resistant Klebsiella pneumoniae. Exp Ther Med 2017;13:1003-10.

27. Micozzi A, Gentile G, Minotti C, et al. Carbapenemresistant Klebsiella pneumoniae in high-risk haematological patients: factors favouring spread, risk 
factors and outcome of carbapenem-resistant Klebsiella pneumoniae bacteremias. BMC Infect Dis 2017;17:203.

28. Machuca I, Gutiérrez-Gutiérrez B, Gracia-Ahufinger I, et al. Mortality Associated with Bacteremia Due to Colistin-Resistant Klebsiella pneumoniae with High-Level Meropenem Resistance: Importance of Combination Therapy without Colistin and Carbapenems. Antimicrob Agents Chemother 2017;61:e00406-17.

29. Papadimitriou-Olivgeris M, Fligou F, Bartzavali C, et al. Carbapenemase-producing Klebsiella pneumoniae bloodstream infection in critically ill patients: risk factors and predictors of mortality. Eur J Clin Microbiol Infect Dis 2017;36:1125-31.

30. Geng TT, Xu X, Huang M, et al. High-dose tigecycline for the treatment of nosocomial carbapenem-resistant Klebsiella pneumoniae bloodstream infections: A retrospective cohort study. Medicine (Baltimore) 2018;97:e9961.

31. Lee NY, Tsai CS, Syue LS, et al. Treatment Outcome of Bacteremia Due to Non-Carbapenemase-producing Carbapenem-Resistant Klebsiella pneumoniae Bacteremia:
Role of Carbapenem Combination Therapy. Clin Ther 2020;42:e33-44.

32. Rivera-Espinar F, Machuca I, Tejero R, et al. Impact of KPC Production and High-Level Meropenem Resistance on All-Cause Mortality of Ventilator-Associated Pneumonia in Association with Klebsiella pneumoniae. Antimicrob Agents Chemother 2020;64:e02164-19.

33. Falcone M, Bassetti M, Tiseo G, et al. Time to appropriate antibiotic therapy is a predictor of outcome in patients with bloodstream infection caused by KPC-producing Klebsiella pneumoniae. Crit Care 2020;24:29.

34. Cooper-Jones B, Farrah K. A Rapid Test for Microbial Identification in Patients With Suspected Sepsis. In: CADTH Issues in Emerging Health Technologies. Ottawa $(\mathrm{ON})$ : Canadian Agency for Drugs and Technologies in Health; September 1, 2017.

35. Du X, Xu X, Yao J, et al. Predictors of mortality in patients infected with carbapenem-resistant Acinetobacter baumannii: A systematic review and meta-analysis. Am J Infect Control 2019;47:1140.
Cite this article as: Qian Y, Bi Y, Liu S, Li X, Dong S, Ju M. Predictors of mortality in patients with carbapenem-resistant Klebsiella pneumoniae infection: a meta-analysis and a systematic review. Ann Palliat Med 2021;10(7):7340-7350. doi: 10.21037/apm21-338 